\title{
Technè
}

La science au service de l'histoire de l'art et de la préservation des biens culturels

41 | 2015

Arts textiles antiques et modernes. Approche scientifique et restauration

\section{Étude d'un paravent du China Trade conservé aux Archives nationales}

Study of a China Trade screen now in France's National Archives

Janin Bechstedt, Patricia Dal-Prà, Hélène Gascuel, Stéphanie Maillet et Sabine Meuleau

\section{(2) OpenEdition}

12 Journals

Édition électronique

URL : http://journals.openedition.org/techne/4891

DOI : 10.4000/techne.4891

ISSN : 2534-5168

Éditeur

C2RMF

Édition imprimée

Date de publication : 25 novembre 2015

Pagination : 108-117

ISBN : 978-2-7118-6248-1

ISSN : $1254-7867$

Référence électronique

Janin Bechstedt, Patricia Dal-Prà, Hélène Gascuel, Stéphanie Maillet et Sabine Meuleau, «Étude d'un paravent du China Trade conservé aux Archives nationales », Technè [En ligne], 41 | 2015, mis en ligne le 08 août 2020, consulté le 11 mars 2021. URL : http://journals.openedition.org/techne/4891 ; DOI : https://doi.org/10.4000/techne.4891

\section{(c)}

La revue Technè. La science au service de l'histoire de l'art et de la préservation des biens culturels est mise à disposition selon les termes de la Licence Creative Commons Attribution - Pas d'Utilisation Commerciale - Pas de Modification 4.0 International. 


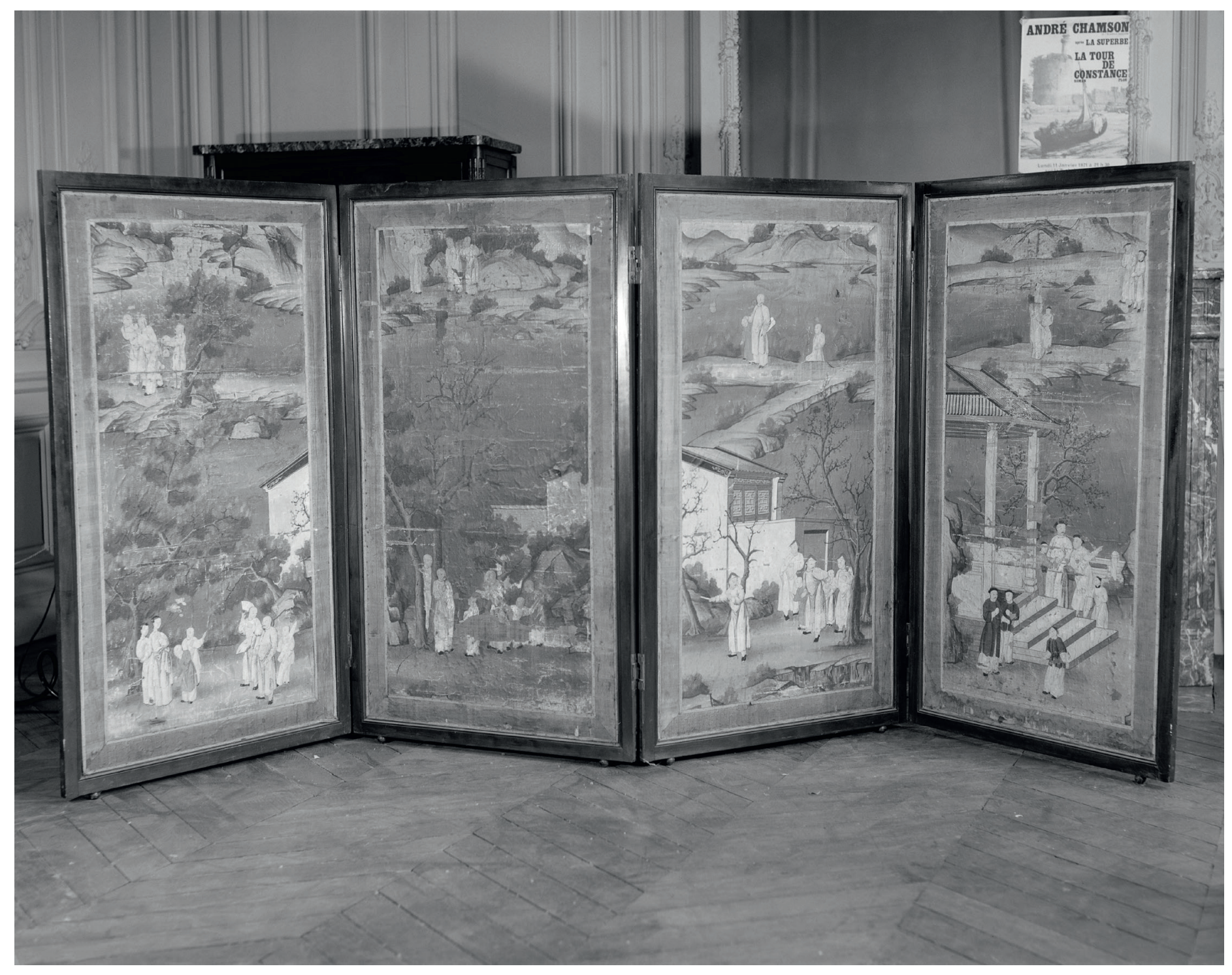

Fig. 1. Vue générale du paravent en 1971. @ Archives nationales, pôle image. 
Janin Bechstedt Patricia Dal-Prà Hélène Gascuel Stéphanie Maillet Sabine Meuleau

\section{Étude d'un paravent du China Trade conservé aux Archives nationales}

Study of a China Trade screen now in France's

National Archives

Résumé. Le musée des Archives nationales conserve un paravent ( $n^{\circ}$ inv. AE/Vla/15) orné de peintures chinoises d'exportation (China Trade) qui porte l'estampille d'un ébéniste parisien de la fin du XVIII siècle. Son état préoccupant a conduit les Archives à commander, en novembre 2012, une étude technique préalable à la restauration accompagnée d'une proposition de traitement. Celle-ci a été confiée à Patricia Dal-Prà et à Janin Bechstedt et a été réalisée dans les ateliers du C2RMF dont le département Recherche a effectué les analyses scientifiques. Elle a été complétée en 2013 par une étude iconographique menée par Hélène Gascuel.

Mots-clés. XVIII siècle, Chine, China Trade, imagerie multispectrale, Marchand-Mercier, Pafrat, Paravent, soie.
Abstract. France's Musée des Archives Nationales has a screen (inventory no. AE/Vla/15), adorned with Chinese paintings for exportation (China Trade), which bears the stamp of a Parisian cabinet-maker active in the late 18th century. Its worrying condition prompted the Archives to commission, in November 2012, a preliminary technical study with a view to its restoration, together with a request for proposals for its treatment. This was entrusted to Patricia Dal-Prà and Janin Bechstedt and carried out in the workshops at C2RMF, whose research department conducted the scientific analyses. It was completed in 2013 by an iconographical study directed by Hélène Gascuel.

Keywords. 18th century, China, China Trade, multispectral imagery, Marchand-Mercier, Pafrat, screen, silk.
Au moment de l'installation des Archives à l'hôtel de Soubise en 1808 , le mobilier des anciens locaux affectés à l'institution (annexes des Assemblées nationales : les Tuileries, puis le Palais-Bourbon) a été déménagé. Les Archives nationales conservent ainsi une série de meubles anciens. La présence d'un paravent dans leurs collections n'a donc rien de surprenant, mais son entrée est peu documentée. Celui-ci est réputé «provenir des Tuileries"sans qu'aucune archive ne vienne étayer cette hypothèse. La première mention de ce paravent apparaît en 1829, dans l'inventaire du mobilier de l'administration des Archives du Royaume ${ }^{1}$ sous la forme d' « un paravent chinois en bois d'acajou, composé de quatre feuilles, couvertes en Pékin à sujets chinois ". L'inventaire de la même série, daté de $1840^{2}$, reprend cette description complétée d'une annotation précisant «mobilier des Tuileries 1792 ». L'état de l'objet est alors décrit comme médiocre. Dans l'inventaire de $1845^{3}$, la mention de provenance des Tuileries est rayée. Elle disparaît des inventaires de 1850 et 1860 et réapparaît, en avril 1885, dans l'inventaire du musée des Archives nationales consacré aux «Objets divers d'origine historique ».

\section{PRÉSENTATION DU PARAVENT}

\section{Description}

Le paravent des Archives nationales est composé de quatre panneaux, ornés chacun au recto et au verso d'une peinture à sujets chinois (fig. 1). Il totalise ainsi huit peintures, de $120 \mathrm{~cm}$ sur $65 \mathrm{~cm}$ environ, exécutées sur satin de soie. Chaque panneau est doublé d'une toile cellulosique grossière, encadré par un ruban bordé de deux galons et monté deux à deux sur un châssis inséré dans un cadre en bois d'acajou vernis. Ce dernier, de facture sobre et élégante, porte au bas du deuxième panneau l'estampille «I. PAFRAT » de l'ébéniste Jean-Jacques Pafrat, reçu maître en 1785 et décédé en $1793^{4}$.

Les panneaux sont numérotés de «I » à « IIII » et des signes « $\Lambda$ » et « V» indiquent le sens d'ouverture des charnières. Chaque panneau est monté sur des roulettes à billes : deux pour les panneaux centraux et une seule pour ceux placés à l'extérieur du paravent. Dans le cadre de notre étude,

Janin Bechstedt, restauratrice de peintures (janin.bechstedt@gmail.com).Patricia Dal-Prà, restauratrice de textiles (patricialdalpra@gmail. com). Hélène Gascuel, conservateur du patrimoine, musée national des Arts asiatiques-Guimet, section Chine (helene.gascuel@guimet.fr). Stéphanie Maillet, chargée de la régie et de la documentation des collections, Département de l'action culturelle et éducative-musée des Archives nationales, Direction des Publics (stephanie.marque-maillet@culture.gouv.fr). Sabine Meuleau, chargée de la valorisation des espaces classés, Département de l'action culturelle et éducative-musée des Archives nationales, Direction des Publics (sabine.meuleau@culture.gouv.fr). 
chaque peinture a reçu un numéro composé du chiffre inscrit au bas du panneau ainsi que de la face, « $\mathrm{A}$ » ou « B », sur laquelle il se trouve.

\section{Production}

\section{Les tentures du China Trade...}

Ces peintures réalisées en Chine relèvent de la production d'exportation à destination des marchés occidentaux, souvent désignée sous le nom de China Trade. Leur réalisation remonte probablement à la seconde moitié du XVIII ${ }^{\mathrm{e}}$ siècle, tandis que l'estampille de Pafrat nous donne un terminus ad quem en 1789, date à laquelle l'ébéniste met fin à son activité.

Bien que ces ouvres soient peintes sur soie, leur format en lés verticaux, de même que leur thème iconographique de figures dans un paysage évoquent les papiers peints chinois d'exportation. Or, si les ouvrages consacrés au China Trade l'oublient trop souvent, certaines des tentures chinoises conservées dans le décor de châteaux européens, à l'instar de celles du château de Dyck situé à une quinzaine de kilomètres au sud-ouest de Düsseldorf, ou du palais de Peterhof dans les environs de Saint-Pétersbourg, sont réalisées sur des lés de soie et non de papier $^{5}$. Les peintures de notre paravent s'inscrivent donc bien dans ce domaine des tentures du China Trade utilisées dans l'ameublement européen du XVIII ${ }^{\mathrm{e}}$ siècle.

L'origine de ce type de tentures remonte à la fin du $\mathrm{XVII}^{\mathrm{e}}$ siècle $^{6}$. Les Chinois n'avaient pas pour habitude d'orner leur intérieur de tentures ou papiers peints. D'après Craig Clunas $^{7}$, les premiers papiers peints, produits en Chine pour l'Europe, seraient nés de la conjonction de deux traditions chinoises : celle de couvrir les murs intérieurs des habitations de feuilles de papier uni et celle de peindre sur de grands formats destinés à être montés aussi bien en rouleaux qu'en écrans.

Les tentures d'exportation étaient produites dans les ateliers du sud de la Chine, à Canton en particulier. Elles sont le fruit d'un travail d'atelier qui impliquait l'intervention de plusieurs artisans spécialisés. Leur support, de papier ou de soie, était apprêté à l'aide d'une préparation de colle et d'alun, avant de recevoir la peinture. Un dessin préparatoire à l'encre était ensuite esquissé à la main - dans de rares cas xylographié - d'après des recueils de modèles, en recourant parfois à la technique du décalque ou du poncif, avant la pose des couleurs. Les tentures et papiers-peints étaient conçus en suite, de sorte qu'ils pouvaient tapisser une pièce entière.

\section{... et leur adaptation en Occident}

Après réalisation, les tentures du China Trade étaient acheminées vers l'Europe et les États-Unis d'Amérique où elles étaient adaptées au décor intérieur des palais et hôtels particuliers, plus rarement aux objets mobiliers.
Les sources nous renseignent sur la vogue des tentures chinoises dans l'ameublement parisien du XVIII ${ }^{\mathrm{e}}$ siècle. En 1753, le Mercure de France publie l'annonce suivante : «On trouvera chez le Sieur Prudhomme, Marchand Papetier, rue des Lombards [...], un assortiment de feuilles de papiers de la Chine de différentes grandeurs, pour tapisseries, dessusde-portes [sic], écrans et paravents ${ }^{8}$. " Cet avis indique que les peintures chinoises sur papier étaient utilisées aussi bien pour tapisser les murs que pour la réalisation d'écrans ou de paravents. Entre 1748 et 1762, le Livre-journal de Lazare Duvaux consigne la livraison d'une vingtaine de paravents en « papier des Indes ${ }^{9}$ ", c'est-à-dire en papier peint chinois. Certains sont décrits de la façon suivante : «paravent à quatre feuilles, en bois d'acajou massif garni de papier des Indes ${ }^{10}$ » ou encore "paravent à cinq feuilles d'acajou garni de très-joli papier des Indes, des deux côtés ${ }^{11}$ »- des termes qui ne sont pas sans évoquer le paravent des Archives nationales réalisé quelques décennies plus tard. L'emploi dans l'ameublement de peintures chinoises sur soie est également attesté dans le Livre-journal de Lazare Duvaux à travers l'expression « toile \& papier des Indes ", qui apparaît une quinzaine de fois sur la même période. Celle-ci ne renvoie cependant jamais à des paravents, mais plutôt à des « châssis » ou " dessus-de-porte » destinés à s'insérer dans les boiseries du décor architectural ${ }^{12}$.

L'intégration des peintures du China Trade au décor intérieur était l'œuvre des tapissiers-décorateurs. Les lés de papier ou de soie étaient doublés de toile et tendus sur un châssis pour être intégrés aux boiseries. Ce montage nécessitait le plus souvent quelques adaptations, de sorte que les peintures étaient couramment retaillées et qu'on leur ajoutait parfois des décors supplémentaires pour emplir les zones vides et dissimuler certains raccords disgracieux ${ }^{13}$. Le paravent des Archives nationales présente un montage de ce type. Son motif indique par ailleurs que les lés ont vraisemblablement été retaillés dans la hauteur. Autant d'éléments qui nous incitent à penser que les peintures qui le garnissent auraient pu avoir été initialement destinées au décor mural. Cependant, leur étroitesse ${ }^{14}$, ainsi que la composition du décor semblent indiquer qu'elles ont probablement bel et bien été conçues dès l'origine pour la garniture d'un paravent. Leur adaptation est l'œuvre d'un ébéniste parisien, travaillant sous la direction d'un marchand-mercier. Importateur et maître d'œuvre, ce «marchand de tout $\&$ faiseur de rien ${ }^{15}$ » constituait un intermédiaire nécessaire entre marchands de la Compagnie des Indes et artisans de divers corps de métier. Ce qui faisait de lui une figure incontournable du commerce et de la transformation des objets exotiques qu'il se chargeait « d'enjoliver » et d'adapter aux besoins et aux goûts de la clientèle occidentale.

\section{Iconographie}

D'un point de vue iconographique, on distingue habituellement deux grandes catégories de tentures chinoises d'exportation : celles à motifs d'arbustes, de fleurs et d'oiseaux d'une 
part et celles représentant des scènes de figures à sujets chinois d'autre part ; toutes deux destinées à satisfaire la soif d'exotisme de la clientèle occidentale. Si la première constitue le groupe le plus important et le plus ancien, nous savons néanmoins que la seconde était de loin la plus recherchée et la plus coûteuse $^{16}$. Particulièrement appréciée pour son pittoresque, elle s'attache à décrire, dans un paysage de campagne, d'architecture ou de jardins, les scènes de la vie chinoise, en privilégiant les activités de loisirs (fêtes, processions et représentations d'acrobates, chasses, promenades et délassements).

Les peintures du paravent des Archives nationales s'inscrivent dans cette veine. Chacun des huit panneaux présente un décor de personnages, distribué sur deux registres au sein d'un paysage continu qui sert de toile de fond à l'ensemble des panneaux. Celui-ci présente néanmoins des ruptures artificielles, fruit d'un montage qui ne respecte pas l'ordre des lés initialement prévu par le peintre chinois. En effet, une observation attentive permet de restituer la séquence originale des panneaux en rendant son unité au paysage (fig. 2).

Il est surprenant de constater que pour retrouver cette séquence, il n'est pas besoin de dissocier les peintures montées deux à deux sur un même cadre. Ceci ne saurait être le fruit d'une simple coïncidence et invite à penser que l'ordre initial des panneaux, scrupuleusement respecté lors du montage des peintures sur leur châssis, a été finalement bouleversé de façon délibérée ou non - au moment de l'assemblage des panneaux en paravent. En effet, à ce stade de notre étude, aucun indice (traces de clous, etc.) ne laisse supposer que le paravent ait pu être démonté et remonté à une date ultérieure et que ce bouleversement puisse donc être postérieur à la date de fabrication.

La scène ainsi restituée se lit de droite à gauche. Elle se déroule dans un paysage composé d'un jardin (avec ses rochers, tables, pavillons et balustrades) au premier plan et d'une campagne lacustre traversée de ponts, avec ses villages et montagnes au loin. On y observe une succession d'événements dont la temporalité est évoquée par le changement des saisons. Celui-ci est indiqué par la végétation, les vêtements - à droite, les hommes portent le chapeau de fonctionnaire d'été, à gauche, celui d'hiver, ainsi que des moufles -, ou encore par les fleurs - le lotus et le chrysanthème font respectivement référence à l'été et à l'automne. Les mêmes personnages semblent se répéter d'une scène à l'autre - à l'instar du jeune garçon identifiable à son habit rouge que l'on retrouve sur la quasi-totalité des panneaux. Leurs mouvements insolites et expressifs pourraient indiquer qu'un épisode narratif - dont la source vraisemblablement littéraire n'a pu être identifiée - sous-tend la représentation. L'ensemble est exécuté avec finesse et force détails, en particulier dans le rendu des vêtements, du mobilier ou du paysage.

\section{ÉTUDE}

Le C2RMF a coordonné l'étude préalable à la restauration. Une fois arrivé dans l'atelier textile du pavillon de Flore, l'ensemble a été photographié et le panneau B1 choisi pour l'étude a été démonté, puis photographié ${ }^{17}$ en imagerie multispectrale - radiographie, réflexion et fluorescence ultraviolettes, lumière du jour, fausses couleurs ultraviolettes et infrarouges, photographie et réflexion infrarouges ${ }^{18}$. Le département Recherche a caractérisé le liant ${ }^{19}$. L'ensemble des informations collectées a contribué à nourrir cette étude qui a impliqué un démontage du panneau peint, la séparation partielle de la soie de la toile de doublage et de nombreux tests de facing, de nettoyage et de retouche.

\section{Examen technologique}

\section{Fibres et tissage}

Le tissu qui sert de support à la peinture est un satin de soie ${ }^{20}$ beige qui comporte ses deux lisières ${ }^{21}$, soit une largeur de lé de $66 \mathrm{~cm}$. Les extrémités supérieures et inférieures sont découpées aux ciseaux.

Une bande de satin de soie de couleur jaune, large de $6 \mathrm{~cm}$, entoure la peinture. Le raccord entre elle et la peinture est caché par un galon de passementerie en soie de couleur jaune de 0,6 cm de largeur. On retrouve le même galon le long du cadre.

Le tissu peint et la bordure jaune sont marouflés sur une toile grossière ${ }^{22}$ en fibre libérienne (chanvre ou jute) écrue. Cette toile comporte une lisière sur un des côtés, tandis que l'autre côté est coupé.

\section{Couche picturale}

\section{Encollage}

La couche picturale est bien assise sur la soie sans pénétration dans les interstices des fils ou des fibres. Ceci indique la présence d'un encollage, ou bien d'un textile peu décreusé, comme c'est souvent le cas pour les soies chinoises destinées à la peinture ${ }^{23}$.

\section{Dessin préparatoire}

À travers la couche picturale et en réflexion infrarouge $(\lambda>750 \mathrm{~nm})$, on distingue la mise en place de la composition. Le dessin préparatoire a été réalisé à l'encre et au pinceau, d'un geste fluide et sûr. Plusieurs détails ont été corrigés au moment de la réalisation définitive ; ainsi dans la corniche du pavillon, dans les cheveux de la première dame à dextre et au niveau du nez du garçon en habit rouge. 
Fig. 2 a. Restitution de la séquence originale des panneaux du paravent.

Face : de gauche à droite (sur la double

page), B4-A3-A1-B2 (assemblage H. Gascuel

et J. Bechstedt). ( ) C2RMF/Elsa Lambert.
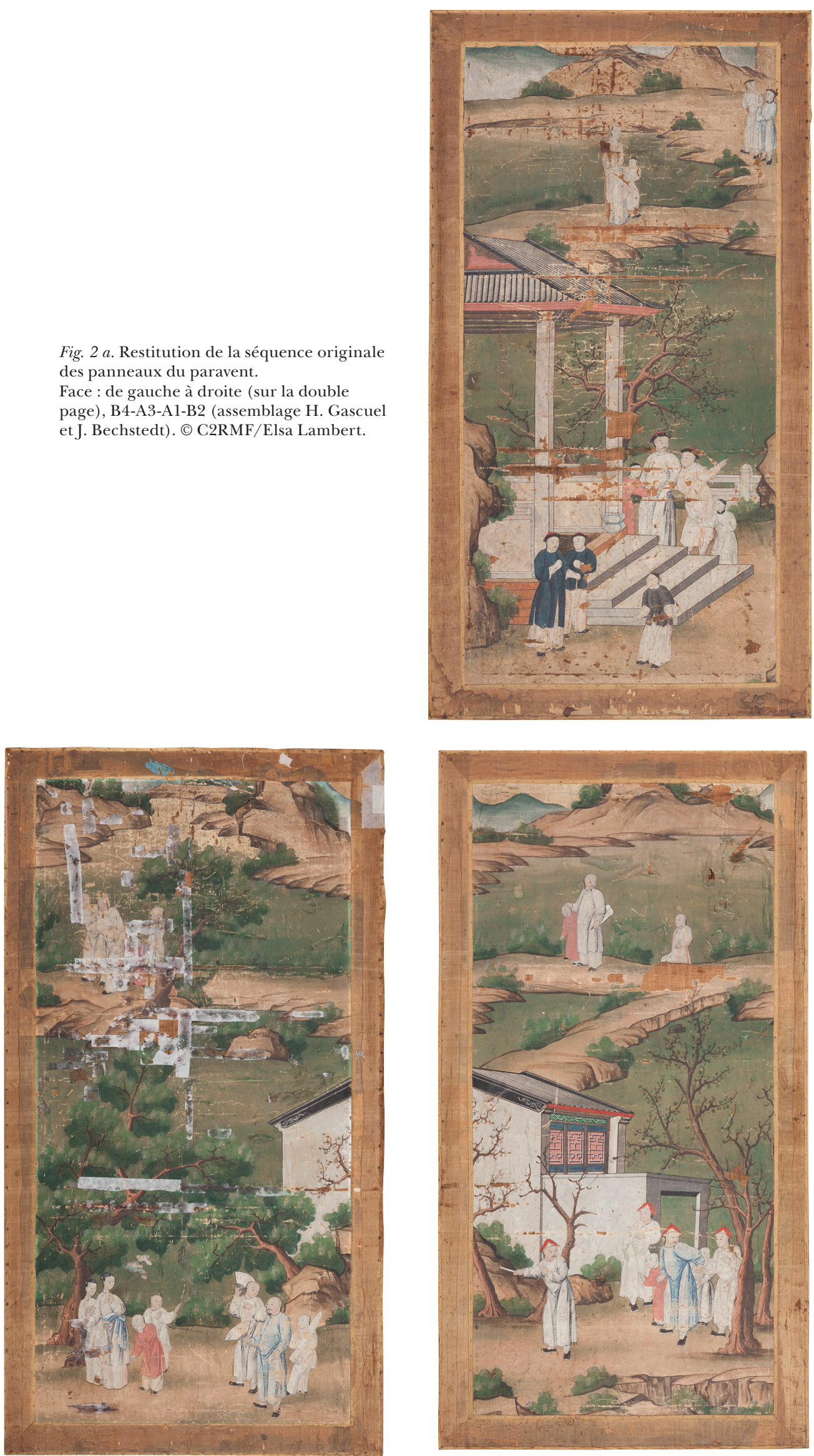

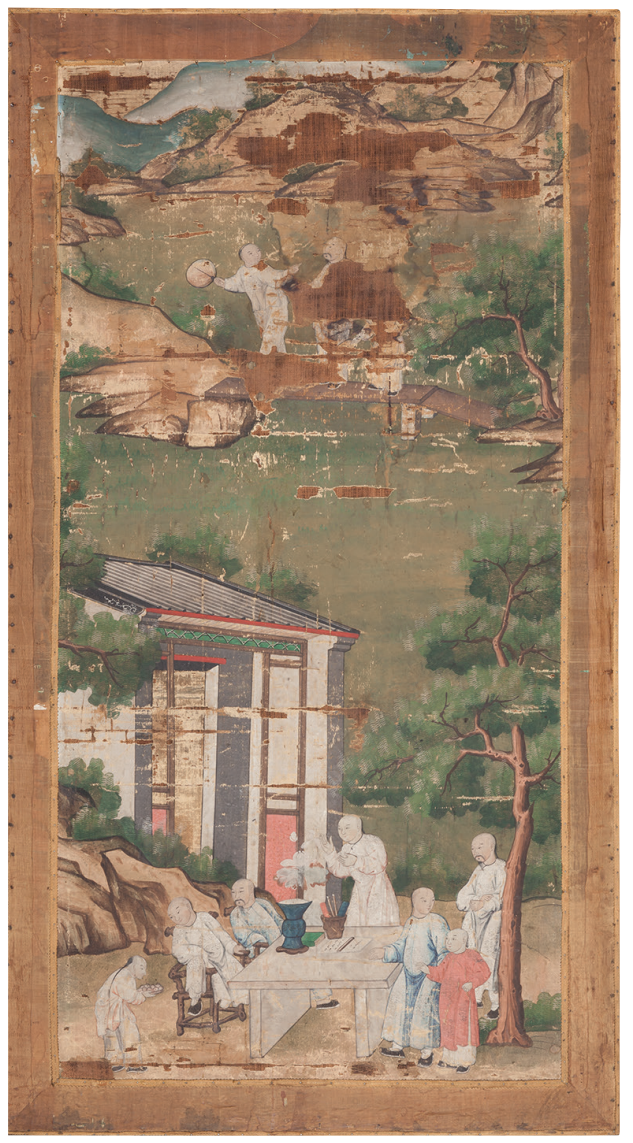



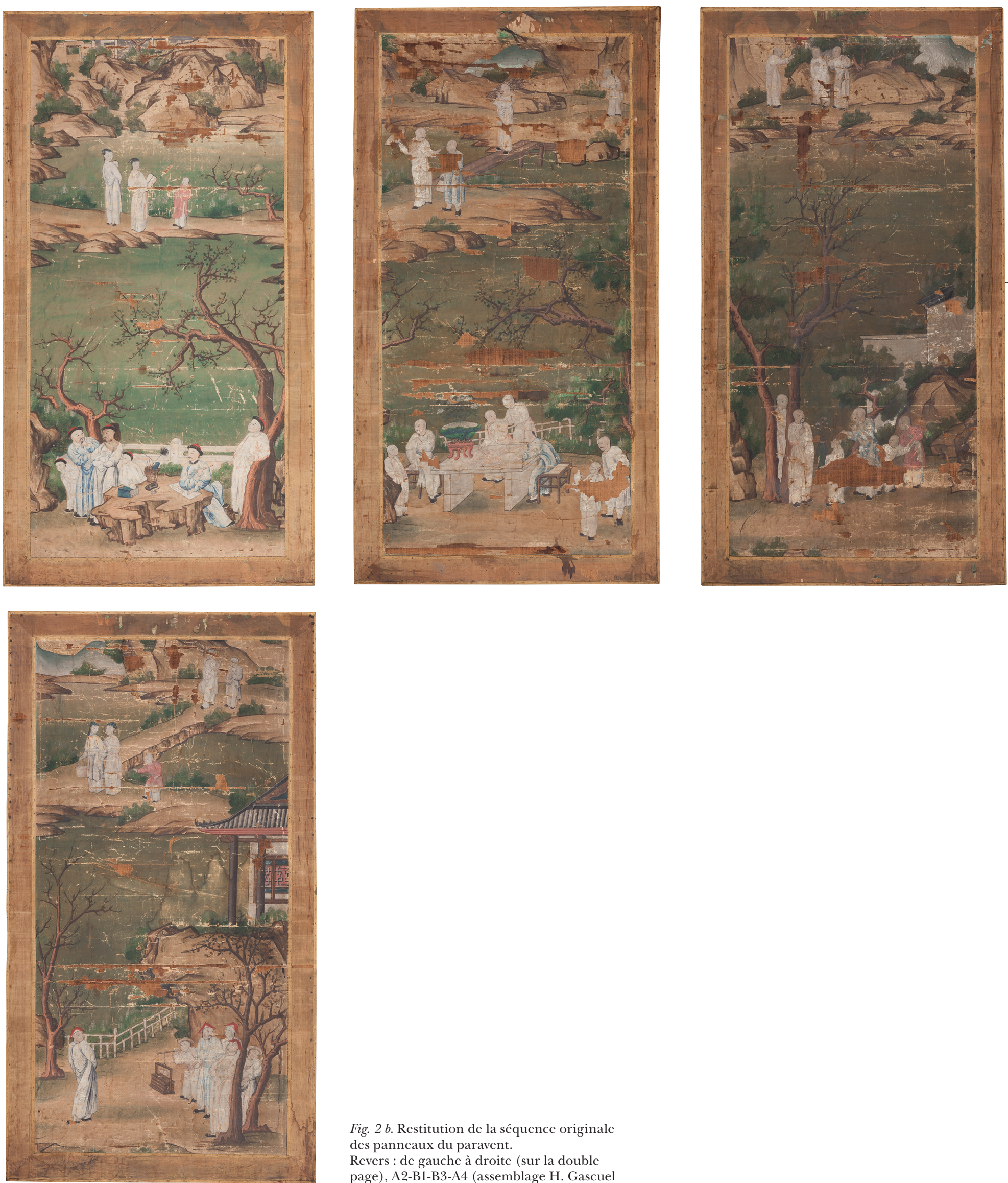

Fig. $2 b$. Restitution de la séquence originale des panneaux du paravent.

Revers : de gauche à droite (sur la double page), A2-B1-B3-A4 (assemblage H. Gascuel et J. Bechstedt). ( $)$ C2RMF/Elsa Lambert. 


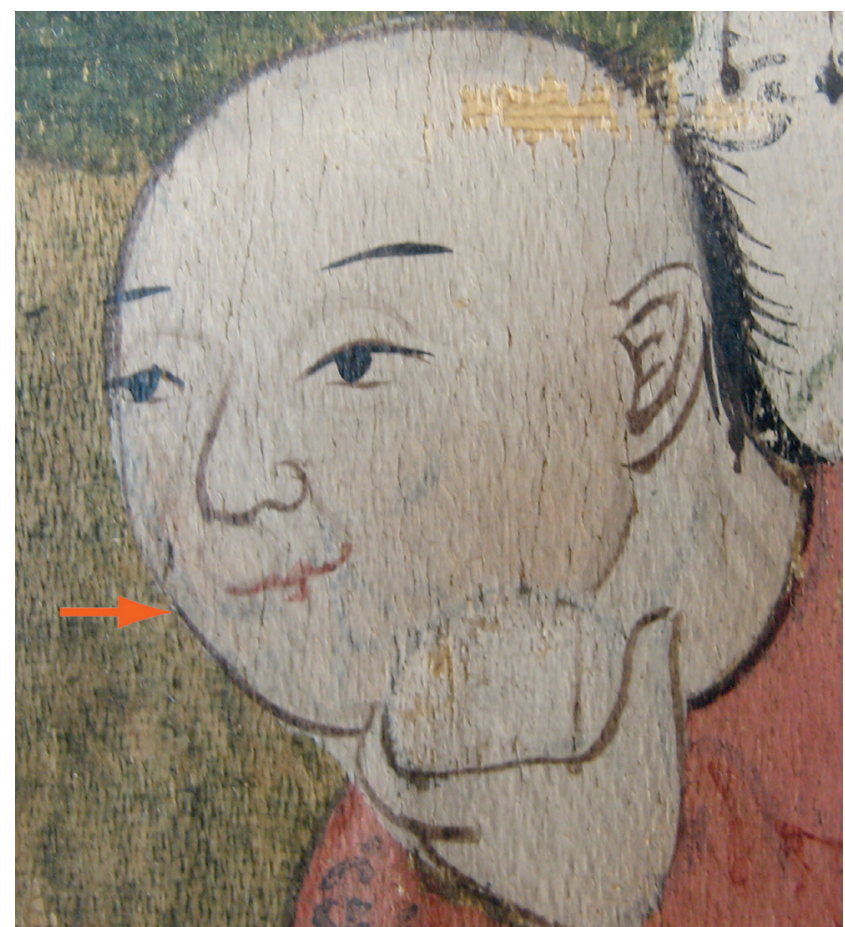

Fig. 3. Repentir sur le nez d'un personnage, détail du panneau B1. (c) Janin Bechstedt.

\section{Couche colorée}

L'artiste a d'abord posé de façon sommaire les arrière-plans, mais en s'avançant vers le spectateur, sa facture devient de plus en plus appliquée et détaillée, même si le modelé et la stratigraphie restent simples. Des zones transparentes ont été juxtaposées à des champs opaques et vice versa. Ainsi, les plages vert clair, assez denses, sont partiellement couvertes d'un jus plus foncé transparent.

Le feuil est fin et lisse et en lumière rasante, on ne distingue aucune trace d'outil. La couche est mate et très poreuse, elle paraît assez souple et présente une sensibilité à l'eau plutôt importante.

Bien que le protocole des prises de vue ait différé de celui des échantillons de référence ${ }^{24}$, les images obtenues dans différentes gammes spectrales ont permis de nombreuses observations, lorsqu'elles ont été couplées à l'examen des couleurs sous microscope binoculaire :

- L’opacité aux rayons X dans les blancs indique la présence d'un élément lourd, très certainement du blanc de plomb.

- Le premier rouge, finement broyé et de couleur chaude, se trouve exclusivement en forme de traits fins dans les plis de certains vêtements blancs. Sa faible quantité en complique l'identification par simple observation sous microscope et dans les différentes longueurs d'ondes. Ces méthodes ont seulement permis de restreindre les nombreux matériaux possibles aux hypothèses d'un colorant, d'un oxyde de fer pur ou d'une terre rouge.

- Le second rouge, mélangé avec du blanc, est visible dans les habits roses du garçon à dextre. Au microscope, on ne distingue pas de grains, ce qui caractérise un colorant organique plus qu'un pigment minéral. L'observation en lumière visible et l'image en fausses couleurs infrarouges indiquent toutes deux la présence d'un colorant.

- Le bleu absorbe considérablement le rayonnement infrarouge, ce qui exclut la présence de l'indigo. Le broyage très fin du pigment et la plupart des images écartent $a$ priori l'hypothèse d'azurite ${ }^{25}$. Il est donc fortement probable qu'un bleu de Prusse ait été employé.

- Au moins quatre verts ont été détectés :

1. Un pigment clair dans les arrière-plans. Son absorption du rayonnement infrarouge, mais surtout son apparence en agrandissement microscopique indiquent la présence d'un minéral contenant du cuivre, très certainement la malachite, finement broyée.

2. Une couleur très proche, mais légèrement plus chaude et plus foncée, située dans les zones claires des buissons. On observe une très forte absorption du rayonnement infrarouge. Sous microscope, on discerne l'apparence spécifique d'un minéral comme la malachite, son broyage est plus grossier que celui du premier vert et elle est peut-être mélangée avec une terre verte pour plus d'opacité.

3. Les jus vert foncé ont été réalisés avec un pigment à granulométrie très fine ou bien un colorant. L'hypothèse d'un mélange de deux colorants jaune et bleu paraît peu probable, les parties protégées de la lumière par le galon présentant la même teinte que certaines parties exposées.

4. Les retouches semblent contenir de l'oxyde de chrome (hydraté aussi bien qu'anhydre) ou du vert de cobalt, car en IR fausses couleurs, on observe une coloration rose et les UV fausses couleurs donnent une coloration brunâtre.

- Les bruns sont vraisemblablement composés de terres (notamment Sienne et Ombre naturelles et brûlées).

- Un pigment à base de carbone a été utilisé pour les noirs.

Étant donné que l'imagerie multispectrale est une méthode d'examen et non d'analyse, les identifications proposées à partir de ces observations et de la microscopie nécessiteront des confirmations par des méthodes complémentaires d'analyse élémentaire ou moléculaire.

Un prélèvement a été réalisé par le C2RMF ${ }^{26}$ sur le bord senestre pour analyser la nature du liant. La chromatographie en phase gazeuse avec spectrométrie de masse a exclu la présence de gommes. Une importante présence de protéines (bien que liée partiellement à la colle de rentoilage), mise en évidence par la coloration spécifique de coupes au Noir Amide, suggère qu'une colle animale pourrait être le liant pictural, ce qui correspond tout à fait à la tradition artistique chinoise. 


\section{Constat d'état}

\section{Textiles}

Le satin de soie peint est très fragilisé. Les flottés du tissage ne sont plus liés et tombent à la moindre pression. Le tissu ne tient plus que par la couche picturale et la toile de doublage. On constate une usure généralisée de la surface davantage marquée au niveau du châssis et tout particulièrement au contact des traverses. Le tissu présente des boursouflures importantes. Il est fendu en de nombreux endroits et ponctuellement lacunaire.

Le satin de soie jaune de l'encadrement adhère étroitement à la toile de doublage. Il a perdu ses flottés et il est lacunaire en de nombreux endroits. On observe clairement la démarcation entre le bord du satin peint découpé irrégulièrement et le reste. Cette différence de niveau a entraîné des ruptures du tissu jaune le long de ce bord. Enfin, il est bruni et porte de nombreuses auréoles et taches, notamment des taches de rouille dues aux clous de fixation.

Le galon est également très fragilisé. Il ne tient plus que par les clous qui le maintiennent au châssis. Sa couleur s'est affadie.

La résistance mécanique de la toile de rentoilage est affaiblie, mais suffisante pour la tension à laquelle elle est soumise à l'intérieur du châssis.

\section{Couche picturale}

Les nombreuses lacunes du support touchent évidemment aussi à la couche picturale. D'autres manques se sont formés dans le sens vertical, suivant les flottés de la soie. Des éraflures et auréoles se situent sur toute la surface. Sur les hauteurs des cloques, la couleur est souvent très usée. Aux endroits où les jus vert foncé s'étalent sur la peinture blanche, ils ont perdu en intensité.

Les traces de colle autour des lacunes ont bruni et les retouches vertes couvrent la peinture d'origine. Toute la surface est fortement altérée par d'importants dépôts de poussière, de crasse et d'excréments d'insectes qui ont pénétré dans les aspérités du feuil et dans le support de soie. Combinées avec un léger jaunissement de la soie, ces substances ont modifié la luminosité et les contrastes de la peinture.

\section{Diagnostic}

La majorité de ces altérations est liée à une exposition trop prolongée au soleil, dont le rayonnement ultraviolet et la chaleur ont accéléré le brunissement et la dégradation physique des soies ainsi que du galon. Parallèlement, les courtes longueurs d'ondes (UV) sont probablement la cause de l'éclaircissement des jus verts par endroits.

L'une des raisons de l'extrême fragilité des fibres de soie pourrait aussi être son exposition aux composés acides dans la toile de doublage ou l'adhésif vieilli. Le marouflage crée
Fig. 4. Jeu de transparence et d'opacité dans la couche picturale, détail du panneau B1. () Janin Bechstedt.

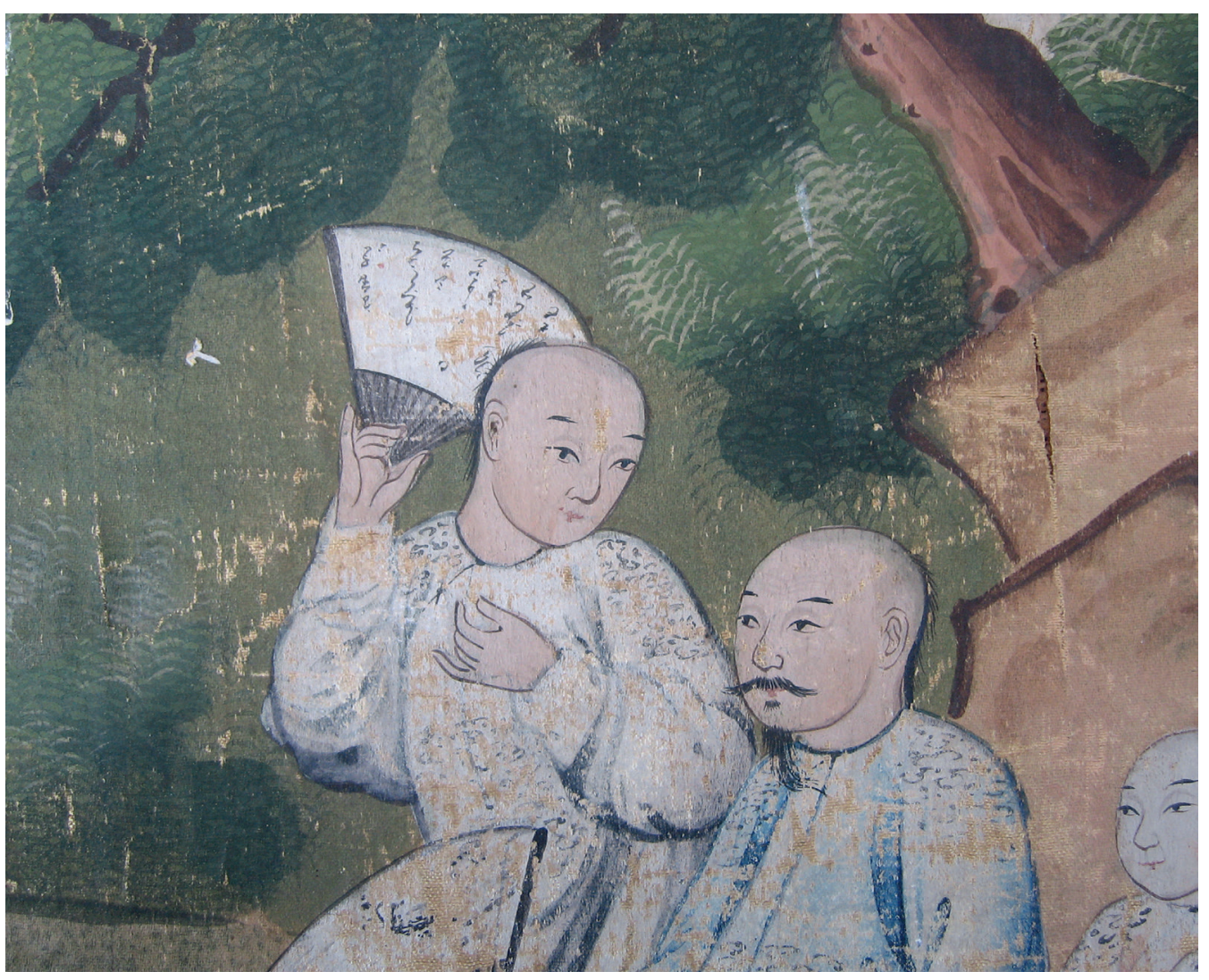


par ailleurs des tensions qui ont entraîné les boursouflures et les fentes jusqu'à une perte de matière.

Une deuxième intervention a alors visé à fixer les bords de certains manques, avec une grande quantité de colle animale trop concentrée qui a bruni en vieillissant. Des retouches très débordantes et dorénavant désaccordées ont peut-être été appliquées au même moment.

L'humidité ambiante a accéléré l'oxydation des clous qui a ensuite altéré le tissu environnant.

À ceci s'ajoutent les auréoles et divers dépôts superficiels généralisés, dont certains comme les déjections d'insectes sont d'une nature extrêmement agressive pour l'œuvre.

\section{Perspectives}

Le paravent des Archives constitue un rare témoin mobilier de l'adaptation des peintures du China Trade aux décors intérieurs européens. Malheureusement, son état préoccupant en interdit aujourd'hui l'exposition au public et compromet très sérieusement son intégrité. Au vu de ce diagnostic, les opérations de restauration suivantes sont prévues :

1. Les peintures seront démontées, une documentation détaillée sera établie et tous les éléments déposés seront conservés à part.

2. Face et revers des panneaux seront dépoussiérés, les repeints seront éliminés et les éventuelles pulvérulences seront refixées.
3. Un facing sera posé sur la couche picturale.

4. La restauration des tissus implique la séparation de ces derniers de la toile de doublage. La méthode qui s'avère la plus efficace et la moins dangereuse pour la couche picturale est celle du dégagement mécanique au moyen d'une lame introduite entre la toile de doublage et l'épaisseur de colle qui la lie à la soie peinte. La colle de rentoilage sera éliminée au maximum pour éviter des réactions hygroscopiques différées.

5. Un rentoilage provisoire sera appliqué et les déformations seront reprises par des tirants en papier ou une mise en extension sur un bâti provisoire.

6. La protection sur la face sera déposée pour procéder au nettoyage, en maîtrisant parfaitement l'apport d'eau. Le satin jaune sera décollé.

7. La face sera de nouveau protégée par un facing, puis les peintures seront retournées pour procéder au doublage définitif. Nous conseillons une technique asiatique traditionnelle à la colle d'amidon et avec des papiers japons, mais l'emploi d'une crêpeline de soie, d'un voile, d'un intissé synthétique ou d'un thermoadhésif peut aussi être envisagé.

8. Après un séchage sous extension, le facing de la peinture sera éliminé pour procéder aux incrustations dans les lacunes dont la longueur est supérieure à environ $0,5 \mathrm{~cm}$. Un textile
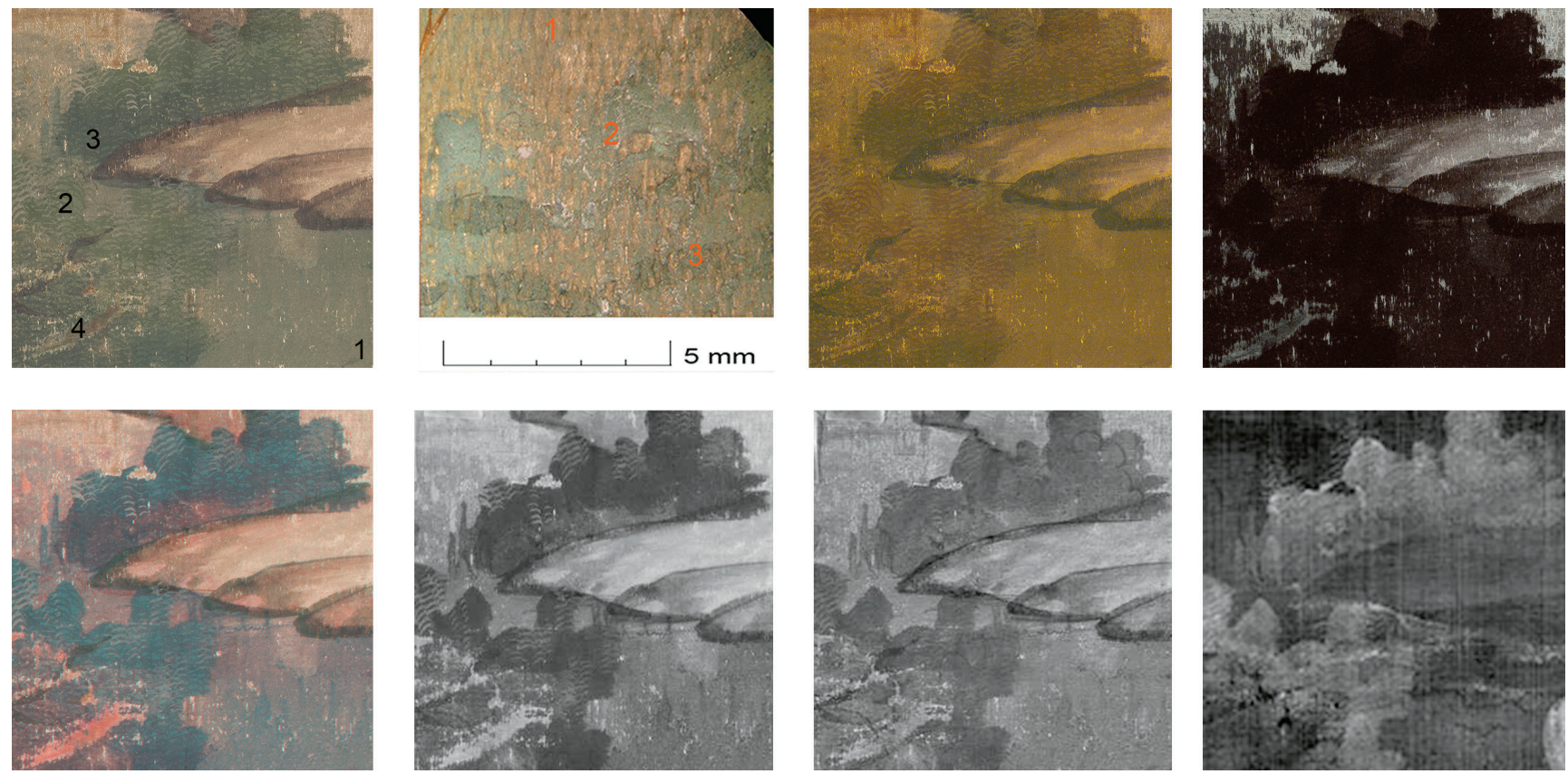

Fig. 5. Détail d'une zone de végétation du panneau B1 visualisé avec différentes méthodes d'imagerie (de gauche à droite et de haut en bas) : lumière visible, agrandissement microscopique, ultraviolet-fausses couleurs, fluorescence, infrarouge-fausses couleurs, photographie infrarouge, réflexion infrarouge, radiographie de rayons $\mathrm{X}$. Les chiffres en rouge correspondent aux pigments mentionnés dans le texte.

(c) C2RMF/Elsa Lambert et Janin Bechstedt. 
avec des fibres, une contexture, un tissage et une couleur au plus proche de l'original sera vieilli artificiellement, afin de diminuer sa résistance mécanique et d'éviter des tensions supplémentaires.

9. De nombreuses lacunes sont d'une étendue trop importante pour une réintégration illusionniste. Nous proposons donc l'approche suivante :

Toutes les lacunes seront retouchées avec un «ton de fond» a tratteggio (imitant la structure du satin) correspondant à leur entourage respectif.

Dans un second temps, la réintégration pourra être avancée en indiquant certaines silhouettes colorées pour les grandes lacunes ou bien en reconstituant de façon presque illusionniste les petits manques.

La réintégration sera réalisée à l'aquarelle avec des pigments résistants au vieillissement.
10. Le panneau sera ensuite remonté sur un carton neutre. Étant donné leur mauvais état de conservation, les bandes de soie jaune et le galon doivent être remplacés. Ce dernier est d'une facture particulièrement fine et sera donc éventuellement retissé d'après l'original.

Après restauration, il faudra également envisager un dispositif sur mesure pour la conservation du paravent qui prendra en considération les exigences spécifiques de l'œuvre et les contraintes liées à son exposition.

Bien que longue et coûteuse, cette restauration présente un intérêt patrimonial et muséographique certain. Elle apportera également de nouvelles données scientifiques qui permettront de mieux caractériser ce paravent et d'élargir ainsi la connaissance des matériaux et techniques employés dans la production du China Trade.
Notes

1. AB XXIII B1, Ch. VII.

2. Ch. XIV.

3. Ch. XIV.

4. Salverte de, p. 253.

5. Wappenschmidt, 1989, p. 33.

6. Wappenschmidt, 1989, p. 26.

7. Clunas et al., 1987, p. 112.

8. Mercure de France dédié au Roi, juin 1753, vol. 2, p. 210.

9. Courajod, 1873, T. 2.

10. Ibid., p. 194.

11. Ibid., p. 382.

12. Courajod, 1873, T. 2.

13. Wappenschmidt, 1989, p. 99-101; Jourdain et Jenyns, 1950, p. 30

14. D'après Wappenschmidt, la largeur des lés de papier-peint chinois varie de 80 à $120 \mathrm{~cm}$, pour une hauteur de $3,50 \mathrm{~m}$ à $4 \mathrm{~m}$. (Wappenschmidt, 1989, p. 32). Au château de Dyck, les lés de la tenture sur soie, datés de la première moitié du XVIII ${ }^{\mathrm{e}}$ siècle, ont une largeur comprise entre 91 et $93 \mathrm{~cm}$. (Wintermann, 2003, p. 92-93).

15. Diderot D. et D'Alembert J. le R.

(dir.), 1751, Tome 10, p. 369

16. Jourdain et Jenyns, 1950, p. 26-27.

17. Par Elsa Lambert (C2RMF)

18. Fausses couleurs ultraviolettes (composite d'une image en lumière visible à $\lambda=400-750 \mathrm{~nm}$ et de la réflexion ultraviolette à $\lambda=350-400 \mathrm{~nm}$ ) et fluorescence (excitation à $\lambda=300-400 \mathrm{~nm}$, enregistrement de l'émission à $\lambda=400-750 \mathrm{~nm})$, photographie infrarouge $(\lambda=700-900 \mathrm{~nm})$, réflexion $(\lambda=900$ $1700 \mathrm{~nm}$ ) et fausses couleurs infrarouges (composite de lumière visible et réflexion infrarouge) et radiographie. Les conditions de prises de vue en infrarouge n'étaient malheureusement pas les mêmes que pour le référentiel, ce qui complique l'identification précise de certains matériaux.

19. Witold Nowik et Myriam Eveno, rapport 28307 du 12.09.2014.

20. Satin de $5:$ satin $=$ une des trois armures fondamentales (toile, sergé, satin), dérivée du sergé ; donne un tissu uni dont les points de liages sont cachés sous de longs flottés (en chaîne ou en trame) ; tout effet de diagonale est évité par la dissémination de ces points de liage ; on ne compte qu'un pris sur chaque fil et sur chaque trame du rapport d'armure, selon un décochement quelconque, qui ne peut toutefois être un diviseur du nombre de fils de l'armure (définition tirée du Vocabulaire technique du CIETA).

21. Armure toile, largeur d'environ $5 \mathrm{~mm}$. $\mathrm{cm}^{2}$.

22. 8 fils de chaîne et 11 fils de trame au

23. Winter, 2008, p. 61.

24. Les prises de vue ont été comparées avec de nombreux échantillons de différentes sources (et donc enregistrés par plusieurs dispositifs), notamment celles de l'Opificio delle Pietre Dure de Florence, d'une recherche effectuée en 2009 par Anne Paounov au C2RMF avec l'École Louis Lumière, ainsi que par Janin Bechstedt lors d'une recherche à l'Institut national du patrimoine en 2010.

25. Nous remercions ici

chaleureusement Annette Keller, photographe et spécialiste en imagerie multispectrale, d'avoir examiné ce détail avec nous.

26. Witold Nowik et Myriam Eveno, rapport $28307 \mathrm{du}$ 12.09.2014.

\section{Références bibliographiques}

Bechstedt J., Papillon M.-C., Vergez P., 2009, "Le secret des trois dames restauration d'une peinture du China Trade et évaluation de l'imagerie en fausses couleurs pour l'identification pigmentaire ", Patrimoines, vol. 5, p. 144-153.

Clunas C. (éd.), 1987, Chinese Export Art and Design, Victoria \& Albert Museum, Londres.
Courajod L. (éd.), 1873, Livre-journal de Lazare Duvaux, marchand-bijoutier ordinaire du Roy (1748-1758) précédé d'une étude sur le goût et sur le commerce des objets d'art au milieu du XVIII ${ }^{e}$ siècle, et accompagné d'une table alphabétique des noms d'hommes, de lieux et d'objets mentionnés dans le journal et dans l'introduction, Paris, 2 vol.

Diderot D. et D'Alembert J. le R. (dir.), 1751, Encyclopédie, Dictionnaire raisonné des sciences, des arts et des métiers, Tome 10, p. 369.

Jourdain M. et Jenyns R. S., 1950, Chinese Export Art in the Eighteenth Century, Country Life Limited, Londres et Charles Scribner's Sons, New York.

Salverte, comte de, 1985, Les ébénistes du XVIII siècle, leurs æuvres et leurs marques, Paris, p. 253.

Wappenschmidt F., 1989, Chinesische Tapeten für Europa. Vom Rollbild zur Bildtapete, Sondereinband, Berlin.

Winter J., 2008, East Asian Paintings Materials, Structures and Deterioration Mechanisms, Archetype Publications, Londres.

\section{Documents inédits}

Paounov A., 2009, L'ultraviolet fausses couleurs : une méthode d'identification des pigments picturaux, vers un protocole de prises de vue destiné à l'ultraviolet, mémoire de fin d'études, École Louis Lumière, Paris, publié sur le site de l'École Louis Lumière.

Wintermann C., 2003, Die handgemalte chinesische Seidentapete auf Papier von Schloss Dyck. Untersuchungen zur Herstellung und Montage, Schadensanalyse. Entwicklung eines Konservierungskonzeptes, mémoire de fin d'études, faculté de Cologne. 